\title{
Femtosecond pump-probe reflectivity study of silicon carrier dynamics
}

\author{
A. J. Sabbah and D. M. Riffe \\ Physics Department, Utah State University, Logan, Utah 84322-4415 \\ (Received 28 May 2002; revised manuscript received 14 August 2002; published 29 October 2002)
}

\begin{abstract}
We have studied the ultrafast optical response of native-oxide terminated $\mathrm{Si}(001)$ with pump-probe reflectivity using $800 \mathrm{~nm}, 28 \mathrm{fs}$ pulses at an excitation density of $(5.5 \pm 0.3) \times 10^{18} \mathrm{~cm}^{-3}$. Time-dependent reflectivity changes comprise third-order-response coherent-transient variations arising from anisotropic state filling and linear-response variations arising from excited free carriers, state filling, and lattice heating. A time constant of $32 \pm 5$ fs associated with momentum relaxation is extracted from the coherent-transient variations. The state-filling and free-carrier responses are sensitive to carrier temperature, allowing an electron-phonon energy relaxation time of $260 \pm 30$ fs to be measured. The recovery of the reflectivity signal back towards its initial value is largely governed surface recombination: a surface recombination velocity of $(3 \pm 1)$ $\times 10^{4} \mathrm{~cm} \mathrm{~s}^{-1}$ is deduced for native-oxide terminated $\mathrm{Si}(001)$.
\end{abstract}

DOI: 10.1103/PhysRevB.66.165217

PACS number(s): 78.47.+p, 42.65.Re, 72.20.-i

\section{INTRODUCTION}

The continuing miniaturization of semiconductor devices into the nanoscale regime has emphasized the importance of understanding carrier dynamics on increasingly shorter time scales. For example, in a nanoscale transistor electron transit times can be a short as a few picoseconds. This time scale is the domain of electron and phonon scattering, and in some cases bulk and surface recombination processes. In order to understand carrier behavior in ever smaller devices, sophisticated Monte Carlo ${ }^{1-4}$ and hydrokinetic transport ${ }^{5}$ models that incorporate both realistic band structure and carrierscattering processes have been developed. A significant result from the hydrokinetic transport theory is that the ratio of carrier momentum-relaxation time to energy-relaxation time has a significant impact on hot-carrier transport. ${ }^{5}$

Ultrafast laser based pump-probe techniques have been instrumental in probing carrier dynamics in crystalline Si. Four techniques have been particularly useful: transient reflectivity (and/or transmission), ${ }^{6-11}$ transient-grating diffraction (including two-beam self-diffraction), ${ }^{12-15}$ photothermal deflection, ${ }^{16,17}$ and time resolved photoemission. ${ }^{18-23}$ In all of these techniques a relatively strong laser pulse (or two crossed pulses in the case of transient grating diffraction) excites valence-band electrons into the conduction band. The relaxation of these electrons and/or the redistribution of the excess energy initially contained in these excited carriers is then monitored with a time-resolved probe. From these studies the following picture of carrier dynamics in $\mathrm{Si}$ has emerged. Initially, momentum-relaxing scattering processes dephase the excited-carrier states on a time scale significantly shorter than $100 \mathrm{fs}^{15,24}$ theoretical calculations suggest that both carrier-carrier ${ }^{25}$ and carrier-phonon ${ }^{3,4,26}$ scattering contribute to the dephasing. Carrier-carrier scattering also produces a well-defined electron temperature (and presumably hole temperature) on a time scale of approximately 100 to $300 \mathrm{fs}^{21,23}$ Electron-phonon scattering also thermalizes the excited carriers with the lattice within a few hundred fs. ${ }^{8,9,14}$ On longer time scales bulk ${ }^{7}$ and surface ${ }^{11}$ recombination processes operate to transfer the excited electrons back to the valence band. At the same time that these local dy- namical processes take place, diffusion ${ }^{10,13}$ and/or bandbending induced transport of the excited carriers may occur. $^{18,19,21,23}$

Here we report on transient reflectivity measurements from $\mathrm{Si}(001)$ using nearly Gaussian $28 \pm 1 \mathrm{fs}$ (FWHM) pulses from a Ti:sapphire oscillator operating at a center wavelength $\lambda=800 \mathrm{~nm}\left(h \nu=E_{\mathrm{ph}}=1.55 \mathrm{eV}\right)$. The small pulse width combined with an excellent signal-to-noise ratio provides a detailed look at the ultrafast dynamics of hot carriers in Si on time scales from the laser pulse width up to our maximum delay time of 120 ps. From the data we obtain quantitative information on carrier momentum relaxation, electron-phonon energy relaxation, and surface mediated electron-hole recombination. This ultrafast work determines a momentum relaxation time in Si. Our measured electronphonon energy relaxation time is combined with earlier measurements of the same quantity at different excess carrier energies. ${ }^{8,9,14}$ The combined experimental results compare favorably with recent Monte Carlo calculations of holeenergy relaxation in $\mathrm{Si}^{4}{ }^{4}$ From the extracted surface recombination velocity we obtain an estimate for the surface defect density of native-oxide terminated $\mathrm{Si}(001)$.

\section{EXPERIMENTAL DETAILS}

The ultrafast pump-probe reflectivity measurements were performed with an in-house constructed Ti-sapphire oscillator, based on the design of Asaki et al. ${ }^{27}$ Interferometric autocorrelation traces obtained with a rotating-mirror autocorrelator are used to determine the laser-pulse widths and confirm the nearly Gaussian nature of the pulses. ${ }^{28}$ Using a half-wave plate and polarizing beam splitter the laser pulses are separated into $p$ - and $s$-polarized pump and probe pulses, respectively. The normally incident $2.80 \pm 0.13 \mathrm{~nJ}$ pump pulses are mechanically chopped and focused to a nearly Gaussian shaped spot with a beam radius $w_{0}=8.5 \mu \mathrm{m}$ and on-axis fluence $f_{c}=2.43 \pm 0.12 \mathrm{~mJ} \mathrm{~cm}{ }^{-2}$. The 33 times weaker probe beam, incident at an angle of $45^{\circ}$, is elliptically shaped (at the sample) with Gaussian beam radii $w_{x}$ $=6.2 \mu \mathrm{m}$ and $w_{y}=4.3 \mu \mathrm{m}$. The reflected probe pulses pass through a linear polarizer, which suppresses scattered light 
from the orthogonally polarized pump, and are detected with a $\mathrm{Si}$ photoconductive detector. The phase-sensitive-detected probe-beam signal (divided by its dc component) is proportional to $\Delta R / R$ ( $R$ is the reflectivity). Most of the runs were obtained with the pump [probe] beam polarization parallel to the (110) $[(1 \overline{1} 0)]$ direction. However, in order to confirm the nonlinear-response nature of the observed initial transient, several runs were also obtained with the sample rotated about its azimuth so that the pump [probe] beam polarization was along the (010) [(100)] direction.

The initially excited carrier density in the near-surface region can be calculated from

$$
N_{0}=\left(1-R_{n}\right) f_{\mathrm{eff}}\left(\frac{\alpha}{E_{\mathrm{ph}}}+\frac{\beta\left(1-R_{n}\right) f_{\mathrm{eff}}}{2 E_{\mathrm{ph}} \tau_{\mathrm{FWHM}}}\right),
$$

where $R_{n}=0.328$ is the normal-incidence reflectivity, ${ }^{29}$ $\tau_{\mathrm{FWHM}}=28 \mathrm{fs}$ is the FWHM pulse width, $f_{\mathrm{eff}}=f_{c}\{[1$ $\left.\left.+\left(w_{x} / w_{0}\right)^{2}\right]\left[1+\left(w_{y} / w_{0}\right)^{2}\right]\right\}^{-1 / 2}=1.76 \pm 0.08 \mathrm{~mJ} \mathrm{~cm}^{-2}$ is the effective incident pump fluence as seen by the probe, $\alpha$ $=1020 \mathrm{~cm}^{-1}$ is the linear absorption coefficient, ${ }^{29}$ and $\beta$ $=6.8 \mathrm{~cm} \mathrm{GW}^{-1}$ is the two-photon absorption-coefficient parameter. We have deduced this value of $\beta$ from previously reported ultrafast measurements with $1.55 \mathrm{eV}, 90$ fs pulses which showed that linear and two photon absorption are equal at $40 \mathrm{~mJ} \mathrm{~cm}^{-2}$ fluence. ${ }^{14}$ Using Eq. (1) we calculate $N_{0}=(5.5 \pm 0.3) \times 10^{18} \mathrm{~cm}^{-3}$.

Measurements were made on a total of four $\mathrm{Si}(001)$ samples, both $n$ and $p$ type with RT carrier concentrations between $10^{13}$ and $10^{15} \mathrm{~cm}^{-3}$. The densities were chosen to be well below the laser excited concentration $N_{0}$ in order to simplify analysis of the reflectivity data. The RT carrier concentration of each sample was determined from the resistivity, ${ }^{30}$ which was measured with a standard four-point probe technique. Surface preparation prior to the resistivity and reflectivity measurements consisted of dipping each sample in concentrated HF acid for two minutes and then rinsing in ultrapure deionized water. A native oxide layer, which has a terminal thickness of $\sim 0.6 \mathrm{~nm}$, was then allowed to grow on each sample by exposure to ambient air for at least $100 \mathrm{~h} .{ }^{31}$ For our experiments this surface oxide is ideal since it is stable in ambient but also thin enough to permit the resistivity measurements to be made.

\section{EXPERIMENTAL RESULTS}

Reflectivity data obtained with 6.67 fs steps are shown in Fig. 1. Data from all four samples are essentially identical to the data shown in the figure. Initially, there is a small, sharp increase in reflectivity (labeled A) followed by a much larger pulse-width limited decrease (B). After this fast decrease the reflectivity continues to decrease, but at a much slower rate (C). The reflectivity then begins to recover approximately $500 \mathrm{fs}$ after the initial excitation (D). As is evident in comparing the data in the lower part of Fig. 1, the initial increase in reflectivity (A) is larger when the pump is polarized along (010) compared to the (110) direction.

As is emphasized in the inset in Fig. 1, data taken with the pump polarized along (110) exhibit a small sinusoidal modu-

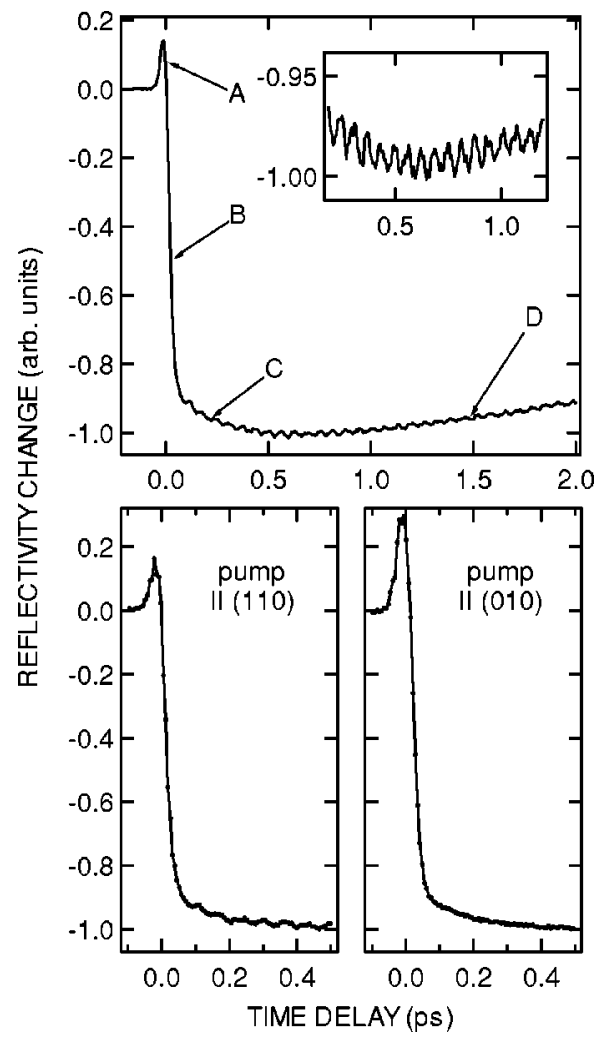

FIG. 1. Time dependent reflectivity change of $\mathrm{Si}$ on a 2-ps time scale. Reflectivity is characterized by a coherent spike (A), pulsewidth limited drop (B), continuing slower drop (C), and subsequent rise (D). Inset illustrates coherent-phonon contribution to reflectivity. Lower part of figure illustrates azimuthal-angle dependence of coherent-spike amplitude.

lation of the reflectivity with a peak-to-peak amplitude in $\Delta R / R$ of $\sim 7 \times 10^{-6}$. This modulation is caused by coherent excitation of the zone-center optical phonon and is discussed in detail elsewhere. ${ }^{32}$

Figure 2 shows data obtained from three of the samples with 120-ps scans. These data indicate the range of variation in recovery of the reflectivity back towards its initial value for the native-oxide terminated samples. Part (b) of Fig. 2 shows the same data as in part (a), with the ordinate expanded to more clearly illustrate the time dependence of the recovery.

\section{CONTRIBUTIONS TO SILICON REFLECTIVITY CHANGES}

In our measurements the $1.55 \mathrm{eV}$ pump pulse excites electrons from states close to the valence band maximum at the Brillouin zone center $(\Gamma)$ to states near the conduction band minimum, which is located at six equivalent $k$-space positions approximately $3 / 4$ of the distance from $\Gamma$ to $X$ [along the (001) and equivalent directions]. Because the pump light is linearly polarized, initial excitation into the six electron pockets is anisotropic. ${ }^{33}$ This anisotropic distribution gives rise to a degenerate four-wave mixing response that is responsible for the initial transient, part A of the reflectivity curve shown in Fig. 1(a). As we show below, this nonlinear 


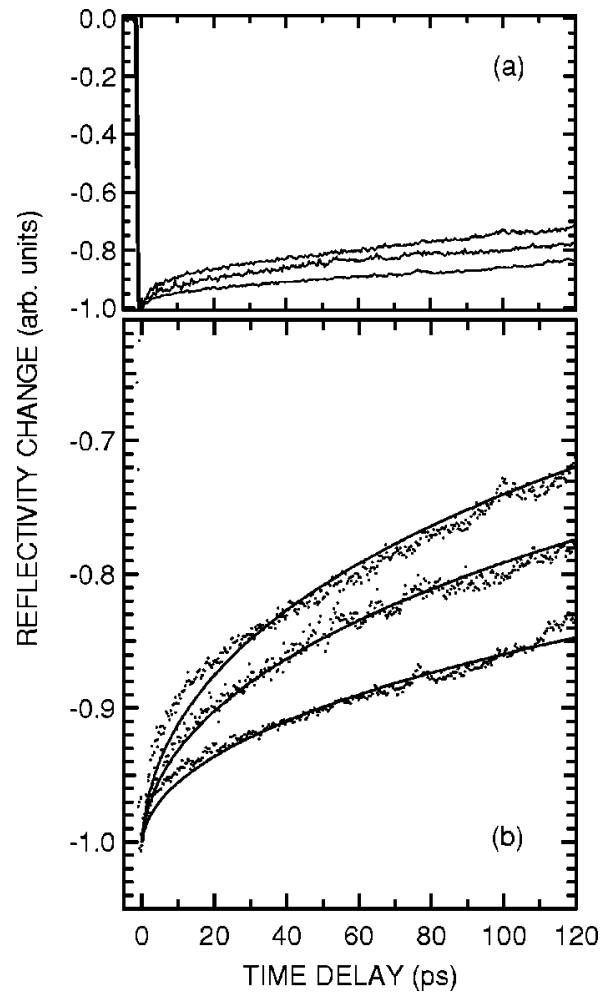

FIG. 2. Time dependent reflectivity of Si on a 120-ps time scale. (a) The three curves illustrate the variation in measured reflectivity for native-oxide covered $\mathrm{Si}(001)$. (b) Measured (dots) and calculated (solid curves) reflectivity changes. The calculated curves correspond to values of $S$ equal to $2.0 \times 10^{4}, 3.4 \times 10^{4}$, and $4.6 \times 10^{4}$ $\mathrm{cm} \mathrm{s}^{-1}$.

optical response contains information on carrier momentum relaxation in Si. The remaining variations in reflectivity are linear-response induced, approximately sequentially due to (1) free-carrier excitation, (2) intraband carrier energy relaxation, and (3) conduction-band to valence-band recombination. As we discuss below, these three processes are responsible for parts $\mathrm{B}, \mathrm{C}$, and $\mathrm{D}$, respectively, of the reflectivity curve in the upper part of Fig. 1. In the subsequent two sections we detail the linear- and nonlinear-response contributions to the reflectivity changes. Our primary goal in these two sections is to obtain a quantitative description of the contributions to the reflectivity changes in order to construct a time-dependent model that can be used to analyze the reflectivity data.

\section{A. Linear-response contributions}

There are two types of linear-response contributions to the measured reflectivity changes: changes in the dielectric function associated with the presence of free-carriers and changes in the dielectric function associated with interband transitions. The free-carrier contribution is commonly described with the Drude model. The interband contribution can arise from three separate effects: state filling, lattice temperature changes, and band-gap renormalization. For our experimental conditions it appears band-gap renormalization changes are insignificant (see discussion at the end of this section).
We thus consider in detail free-carrier, state-filling, and lattice-temperature contributions to the reflectivity.

In general, changes in reflectivity of a material can be related to changes in the dielectric function. The preexcitation, room-temperature dielectric function of $\mathrm{Si}$ at $800 \mathrm{~nm}$ is $\hat{\varepsilon}_{0}=13.656+i 0.048 .{ }^{29}$ However, because $\operatorname{Re}\left(\hat{\varepsilon}_{0}\right) \gg \operatorname{Im}\left(\hat{\varepsilon}_{0}\right)$ and for our experimental conditions $|\Delta \hat{\varepsilon}| \ll\left|\hat{\varepsilon}_{0}\right|$ and $\operatorname{Re}(\Delta \hat{\varepsilon}) \geq \operatorname{Im}(\Delta \hat{\varepsilon}),(\Delta \hat{\varepsilon}$ is the change in the dielectric function) we only need to consider $\operatorname{Re}(\Delta \hat{\varepsilon})$ in calculating or modeling the reflectivity changes. In terms of the complex index of refraction $\hat{n}=n+i \kappa=(\hat{\varepsilon})^{1 / 2}$ this is equivalent to only considering the contribution of $\Delta n$ to the reflectivity variations. Under these conditions the normalized change in $s$-polarized reflectivity $R_{s}$ is related to $\Delta n$ with very little loss of accuracy $(<0.1 \%)$ via

$$
\frac{\Delta R_{s}}{R_{s}}=\frac{4 n_{0} \cos (\theta)}{\left(n_{0}^{2}-1\right)\left[n_{0}^{2}-\sin ^{2}(\theta)\right]^{1 / 2}} \Delta n
$$

where $\theta$ is the angle of incidence and $n_{0}=\operatorname{Re}\left(\hat{\varepsilon}_{0}^{1 / 2}\right)$. Because the free-carrier (FC), state-filling (SF), and latticetemperature (LT) contributions to $\Delta n$ are all much smaller than $n$ we can write the total change in refractive index as $\Delta n=\Delta n_{\mathrm{FC}}+\Delta n_{\mathrm{SF}}+\Delta n_{\mathrm{LT}}$.

Both the free-carrier and state-filling contributions to the reflectivity depend upon the time dependence of the excited carrier density $N$ and carrier temperature $T_{e}$. The carrier density $N$ first increases due to pump-pulse excitation and then decreases via recombination. As is evident below from the data analysis, at short times the recombination contribution to the time dependence of $N$ is described very well by exponential decay (effective relaxation time $\equiv \tau_{R}$ ) to a nonzero background. That is, for an initial excitation density $N_{\text {in }}$ the short-time time dependence of $N$ can be expressed as

$$
N(t)=N_{\mathrm{in}} \frac{1+C_{R} \exp \left(-t / \tau_{R}\right)}{1+C_{R}},
$$

where the constant $C_{R}$ defines the nonzero background for the decay.

Just after initial excitation the carriers each have an average excess energy of $0.30 \mathrm{eV}$ (above the band edge). ${ }^{34}$ As discussed in detail in Sec. V. A, this excess energy becomes thermalized among the carriers on a time scale that is significantly longer than the laser pulse width, so that a true carrier temperature only develops on a time scale comparable to the carrier-lattice equilibration time. However, for simplicity in our analysis we assume that the carriers can be described by an effective temperature. For $\mathrm{Si}$ the initial excess carrier energy of $0.3 \mathrm{eV}$ corresponds to a hot-carrier temperature of $1985 \mathrm{~K}^{35}$ The hot carriers equilibrate with the lattice with a time constant $\tau_{\mathrm{el} \text {-ph }}$-known as the electron-phonon energy relaxation time-which is on the order of a few hundred fs. ${ }^{8,9,14}$ The carriers and lattice equilibrate very close to RT because of the relatively small number of carriers that are excited. The electron-phonon energy relaxation time is only a very weak function of excess carrier temperature; ${ }^{8,9,14}$ hence, 
for an initial carrier temperature $T_{\text {in }}$ the time dependence of the carrier temperature can be written as

$$
T_{e}(t)=\left(T_{\text {in }}-300 \mathrm{~K}\right) \exp \left(-t / \tau_{\text {el }-p h}\right)+300 \mathrm{~K} .
$$

Using the Drude model, the free carrier contribution $\Delta n_{\mathrm{FC}}$ to $\Delta n$ can be expressed as ${ }^{6}$

$$
\Delta n_{\mathrm{FC}}\left(N, T_{e}\right)=-\frac{2 \pi e^{2}}{n_{0} \omega^{2}} \frac{N}{m_{\mathrm{opt}}^{*}\left(T_{e}\right)},
$$

where $\omega$ is the (angular) laser frequency, $e$ is the electron charge, and $m_{\mathrm{opt}}^{*}\left(T_{e}\right)=\left(1 / m_{e}^{*}+1 / m_{h}^{*}\right)^{-1}$ is the carrier effective optical mass. Here $m_{e}^{*}$ and $m_{h}^{*}$ are the electron and hole conductivity effective masses, respectively. The $T_{e}$ dependence of $m_{\mathrm{opt}}^{*}$ arises from band nonparabolicity. ${ }^{36}$ Recently we calculated the temperature dependences of $m_{e}^{*}$ and $m_{h}^{*}$, and thus $m_{\text {opt }}^{*}{ }^{37}$ The calculation yields $m_{\text {opt }}^{*}(1985 \mathrm{~K})$ $\equiv m_{1985}^{*}=0.205 m_{e}$ and $m_{\mathrm{opt}}^{*}(300 \mathrm{~K}) \equiv m_{300}^{*}=0.156 m_{e}\left(m_{e}\right.$ is the free electron mass). With $N=N_{0}$, Eq. (5) yields $\Delta n_{\mathrm{FC}}\left(N_{0}, 1985 \mathrm{~K}\right)=-2.1 \times 10^{-3}$ and $\Delta n_{\mathrm{FC}}\left(N_{0}, 300 \mathrm{~K}\right)=$ $-2.8 \times 10^{-3}$. Thus, the free carrier response initially produces a negative contribution to the reflectivity change, and as the carriers cool the negative change increases in magnitude. At longer times, when recombination becomes important, the decrease in $N$, [Eq. (3)], serves to decrease the freecarrier contribution to the reflectivity change.

Theoretical results for $m_{\mathrm{opt}}^{*}\left(T_{e}\right)$ at other temperatures are summarized in Fig. 3, which shows that $m_{\mathrm{opt}}^{*}\left(T_{e}\right)$ increases nearly linearly vs $T_{e}$ above RT. Therefore $m_{\mathrm{opt}}^{*}\left(T_{e}\right)$ exponentially decays to its RT value $m_{300}^{*}$ with the time constant $\tau_{\text {el-ph }}$. Putting together the expected time dependences of $N$ and $m_{\mathrm{opt}}^{*}$, we obtain a time dependent free-carrier response function (defined for $t>0$ )

$$
\begin{aligned}
A_{\mathrm{FC}}(t)= & -A_{\mathrm{FC}}^{0} \frac{1}{1+\left(\Delta m_{\mathrm{opt}}^{*} / m_{300}^{*}\right) \exp \left(-t / \tau_{\mathrm{el}-\mathrm{ph}}\right)} \\
& \times \frac{1+C_{R} \exp \left(-t / \tau_{R}\right)}{1+C_{R}},
\end{aligned}
$$

where $\Delta m_{\mathrm{opt}}^{*}=m_{1985}^{*}-m_{300}^{*}$ and $A_{\mathrm{FC}}^{0}$ is a positive constant.

The free-carrier, time-dependent contribution to the measured reflectivity change is calculated from $A_{\mathrm{FC}}(t)$ via

$$
\begin{aligned}
\frac{\Delta R_{\mathrm{FC}}}{R_{s}}(\Delta t)= & \int_{-\infty}^{\infty} d t\left|E_{x}^{(p)}(t-\Delta t)\right|^{2} \\
& \times \int_{-\infty}^{t} d t^{\prime} A_{\mathrm{FC}}\left(t-t^{\prime}\right)\left|E_{y}^{(e)}\left(t^{\prime}\right)\right|^{2} .
\end{aligned}
$$

Here $E_{y}^{(e)}(t)$ and $E_{x}^{(p)}(t)$ are the (envelope) electric fields associated with the excitation (pump) and probe pulses, respectively, and $\Delta t$ is the time delay between these two pulses. In our experiment the excitation and probe electric fields are well approximated by the Gaussian function $E(t)$ $=E_{0} \exp \left[-\left(t / \tau_{p}\right)^{2}\right]$, where $\tau_{p}$ is related to the pulse intensity FWHM $\tau_{\text {FWHM }}$ via $\tau_{p}=\tau_{\text {FWHM }} / 2 \ln (2)=24 \pm 1$ fs. (In our ex-

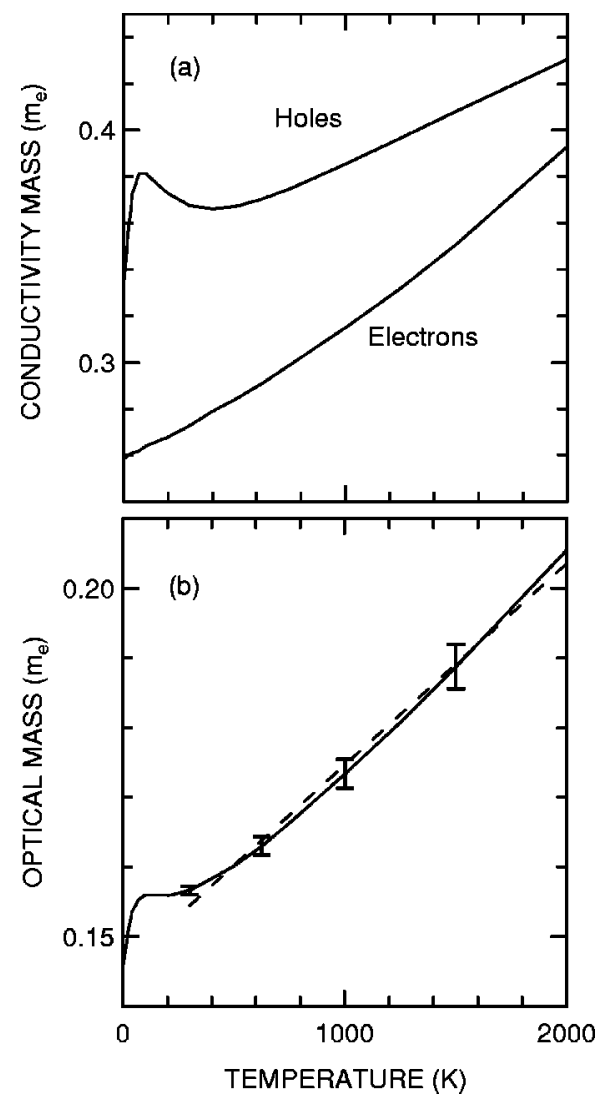

FIG. 3. Calculated carrier effective masses versus temperature. (a) Conductivity effective masses of holes and electrons. (b) Optical effective mass. The dashed line is a linear fit to the calculation between RT and $2000 \mathrm{~K}$.

periment the noncolinearity and finite spot sizes of the pump and probe beams introduce a small correction that results in a slightly larger effective $\tau_{p}$. For our experimental geometry we calculate a $6 \%$ correction factor, which results in an effective $\tau_{p}=25 \pm 1$ fs.) Using this form for $E(t)$, Eq. (6) for $A_{\mathrm{FC}}(t)$, and the relationships $\tau_{p} \ll \tau_{\text {el-ph }}, \tau_{p} \ll \tau_{R}$, Eq. (7) can be expressed, to very good approximation, as

$$
\begin{aligned}
& \frac{\Delta R_{\mathrm{FC}}}{R_{s}}(\Delta t)=\frac{\Delta R_{\mathrm{FC}}^{\max }}{R_{s}} \frac{\left[\operatorname{erf}\left(\Delta \mathrm{t} / \tau_{\mathrm{p}}\right)+1\right] / 2}{1+\left(\Delta m_{\mathrm{opt}}^{*} / m_{300}^{*}\right) \exp \left(-\Delta t / \tau_{\text {el-ph }}\right)} \\
& \times \frac{1+C_{R} \exp \left(-\Delta t / \tau_{R}\right)}{1+C_{R}}
\end{aligned}
$$

The term $\Delta R_{\mathrm{FC}}^{\max } / R_{s}$ is the Drude contribution to the change in reflectivity for $N=N_{0}$ and $T_{e}=300 \mathrm{~K}$.

Pump-pulse excitation also modifies the interband dielectric function due to redistribution of electrons from the valence to conduction band: after excitation there are fewer electrons that can be excited from the valence band and fewer empty states in the conduction band that can be filled. In general this state filling effect decreases the absorption coefficient $\alpha(=4 \pi \kappa / \lambda)$ for photon energies above the band 
gap. Since the excited electron and hole distributions depend upon $N$ and $T_{e}$, the change in $\alpha$ due to state filling also depends on $N$ and $T_{e}$, i.e., $\Delta \alpha_{\mathrm{SF}}=\Delta \alpha_{\mathrm{SF}}\left(N, T_{e}\right)$. Via a Kramers-Kronig relationship the change in the real part of the index of refraction $\Delta n_{\mathrm{SF}}\left(N, T_{e}\right)$ can be calculated from the photon energy $\left(E_{\mathrm{ph}}\right)$ dependence of $\Delta \alpha_{\mathrm{SF}}\left(N, T_{e}\right) .{ }^{38} \mathrm{In}$ order to estimate $\Delta \alpha_{\mathrm{SF}}\left(N, T_{e}\right)$ and $\Delta n_{\mathrm{SF}}\left(N, T_{e}\right)$, we assume that $\alpha=K_{\alpha}\left(E_{\mathrm{ph}}-E_{g}\right)^{2}$, which is theoretically expected for indirect (phonon assisted) absorption between parabolic bands ${ }^{39}$ and is observed for $\mathrm{Si}$ for photon energies between 1.5 and $2.1 \mathrm{eV}^{29}$ Here $K_{\alpha}$ is a constant (which we have determined by fitting absorption coefficient data ${ }^{29}$ ), and $E_{g}$ $=1.12 \mathrm{eV}$ is the indirect RT band gap of $\mathrm{Si}$.

With this model we have calculated $\Delta \alpha_{\mathrm{SF}}\left(N, T_{e}\right)$ and $\Delta n_{\mathrm{SF}}\left(N, T_{e}\right)$. The calculated value for $\Delta \alpha_{\mathrm{SF}}\left(N, T_{e}\right)$ at $E_{\mathrm{ph}}$ $=1.55 \mathrm{eV}$ is expected to be quite accurate since $1.55 \mathrm{eV}$ is in the range where $\alpha=K_{\alpha}\left(E_{\mathrm{ph}}-E_{g}\right)^{2}$ is valid. However, because the Kramers-Kronig calculation of $\Delta n_{\mathrm{SF}}\left(N, T_{e}\right)$ depends upon values of $\Delta \alpha_{\mathrm{SF}}\left(N, T_{e}\right)$ at photon energies much greater than $E_{\mathrm{ph}}=1.55 \mathrm{eV}$ where $\alpha=K_{\alpha}\left(E_{\mathrm{ph}}-E_{g}\right)^{2}$ no longer holds, the calculated values of $\Delta n_{\mathrm{SF}}\left(N, T_{e}\right)$ are only semiquantitative in nature. For $N=N_{0}$ the model yields $\Delta n_{\mathrm{SF}}\left(N_{0}, 1985 \mathrm{~K}\right)=-5.0 \times 10^{-4}$ and $\Delta n_{\mathrm{SF}}\left(N_{0}, 300 \mathrm{~K}\right)=$ $-2.4 \times 10^{-4}$. Although these values are only semiquantitative, we expect that the negative sign associated with $\Delta n_{\mathrm{SF}}\left(N, T_{e}\right)$ and the increase in magnitude with temperature to be robust results. Thus, state filling also produces an initially negative contribution to the reflectivity change. However, in contrast to the free-carrier contribution the negative change becomes smaller in magnitude as the carriers cool to RT. Comparing the Drude-model calculations of $\Delta n_{\mathrm{FC}}\left(N_{0}, T_{e}\right)$ vs $T_{e}$ and these estimations for $\Delta n_{\mathrm{SF}}\left(N_{0}, T_{e}\right)$ vs $T_{e}$, we expect the (negative) free-carrier change to dominate the (positive) state-filling change as the carriers cool. Thus the calculations indicate that the reflectivity should continue to decrease as the carriers cool. This is in agreement with the experimental observation of a continued decrease in reflectivity up to a time delay of $\sim 500 \mathrm{fs}$ as shown in part C of the reflectivity curve in Fig. 1.

In order to derive a response function due to state-filling we note that our calculation of $\Delta \alpha_{\mathrm{SF}}\left(N, T_{e}\right)$ is also quite linear as a function of $T_{e}$ between 300 and $2000 \mathrm{~K}$. With the assumption that $\Delta n_{\mathrm{SF}}\left(N, T_{e}\right)$ is also linear as a function of $T_{e}$, we obtain a state-filling response function of the form

$$
\begin{aligned}
A_{\mathrm{SF}}(t)= & -A_{\mathrm{SF}}^{0}\left[1+\left(\frac{\Delta n_{1985}}{\Delta n_{300}}-1\right)\right. \\
& \left.\times \exp \left(-t / \tau_{\mathrm{el}-\mathrm{ph}}\right)\right] \frac{1+C_{R} \exp \left(-t / \tau_{R}\right)}{1+C_{R}},
\end{aligned}
$$

where $A_{\mathrm{SF}}^{0}$ is a positive constant and $\Delta n_{T} \equiv \Delta n_{\mathrm{SF}}\left(N_{0}, T\right)$. Here we have also assumed that the response function is proportional to $N$, which is valid at nondegenerate excitation densities $\left(N \leqq 10^{20} \mathrm{~cm}^{-3}\right)$. Analogous to Eq. (8) for the freecarrier contribution to $\Delta R_{s} / R_{s}$, the state filling contribution can be written as

$$
\begin{aligned}
\frac{\Delta R_{\mathrm{SF}}}{R_{s}}(\Delta t)= & \frac{\Delta R_{\mathrm{SF}}^{\max }}{R_{s}} \frac{\operatorname{erf}\left(\Delta t / \tau_{p}\right)+1}{2}\left[1+\left(\frac{\Delta n_{1985}}{\Delta n_{300}}-1\right)\right. \\
& \left.\times \exp \left(-\Delta t / \tau_{\mathrm{el}-p h}\right)\right] \frac{1+C_{R} \exp \left(-\Delta t / \tau_{R}\right)}{1+C_{R}} .
\end{aligned}
$$

The lattice-temperature contribution to the reflectivity variations can be deduced from experimental measurements of $d n / d T_{L}$. We use $d n / d T_{L}=3.4 \times 10^{-4} \mathrm{~K}^{-1}$, which we have obtained by extrapolating measured values of $d n / d T_{L}$ at wavelengths between 450 and $750 \mathrm{~nm} .{ }^{40}$ We calculate the rise in lattice temperature due to the excited electrons and holes relaxing to their respective band edges to be 0.30 $\pm 0.02 \mathrm{~K}$. Using

$$
\Delta n_{\mathrm{LT}}=\frac{d n}{d T_{L}} \Delta T_{L}
$$

this rise in temperature produces $\Delta n_{\mathrm{LT}}=1.0 \times 10^{-4}$. Thus, a rise in lattice temperature serves to increase the reflectivity. However, the lattice-temperature contribution is negligible compared to the free-carrier and state-filling contributions, at least before recombination becomes important. We thus neglect this contribution in analysis of the short-time reflectivity. At longer times when recombination dominates the dynamics and the band-gap energy is transferred back to the lattice with each recombination event, the lattice-temperature contribution to the reflectivity can become significant. Thus in analyzing the 120-ps scans (see Sec. V B) we include the lattice-temperature contribution.

Any contribution to the reflectivity changes from bandgap renormalization is rather difficult to quantify. In principle, at high enough excitation densities carrier many-body effects can effectively reduce the optical band gap, resulting in a change in dielectric function that is equivalent to an increase in the photon energy. ${ }^{41-43}$ For $\mathrm{Si}$ this would produce a positive change in $n \cdot{ }^{29}$ However, this effect is significantly different than the effects discussed above in that (1) there is expected to be a critical carrier density $N_{\text {cr }}$ (slightly larger than the Mott density) below which band-gap renormalization is absent, ${ }^{41}$ and (2) for densities above $N_{\text {cr }}$ the change in refractive index is expected to slowly vary with $N$, typically as $N^{1 / 3}$ or $N^{1 / 4} .{ }^{44}$ Using the expression of Bennett et al., ${ }^{41}$ we calculate $N_{\mathrm{cr}}=6 \times 10^{18} \mathrm{~cm}^{-3}$, slightly above our excitation density. Thus, the effect of band-gap renormalization on the reflectivity may not be significant. Further, at excitation densities close to or significantly exceeding $N_{\mathrm{cr}}$, prior ultrafast experiments on Si show no evidence of a band-gap renormalization contribution to the change in optical constants. ${ }^{6,9,10,17}$ We thus adopt the viewpoint that any band-gap renormalization contribution to the reflectivity is minimal and can thus be neglected in modeling our measured reflectivity changes.

We note that in analyzing our experimental results nearsurface values of $\Delta n$ can be used. This is because for our experimental conditions the spatial variation of $\Delta n$ is nearly 
always much greater than the effective sampling depth of $\lambda /\left(4 \pi\left|\hat{n}_{0}\right|\right)=17 \mathrm{~nm}$. This is discussed in detail in the Appendix.

\section{B. Coherent-transient contributions}

The initial transient spike observed in our measurements is similar to coherent transients previously observed using other pump-probe experimental geometries that are also sensitive to degenerate four-wave mixing in semiconductors. These geometries include transmission, ${ }^{45}$ two-beam self-diffraction, ${ }^{15,46}$ and reflective transient-grating diffraction. ${ }^{14,24}$

In our discussion of coherent transient effects we are guided by the degenerate four-wave mixing theory of Smirl and co-workers. ${ }^{47,48}$ There are two contributions to the change in intensity in the (reflected) probe-beam direction. The first contribution arises from pump light that is diffracted from the polarization grating that is formed by the overlap of the (orthogonally polarized) pump and probe beams at the sample surface. This polarization grating arises from the anisotropic momentum distribution of the initially excited carriers. The anisotropy decays via carrier-carrier and carrier-phonon scattering on a timescale that is significantly less than $100 \mathrm{fs}{ }^{15,24}$ Because this momentum randomization is significantly faster than the other relaxation processes (energy relaxation, recombination, and diffusion) that govern the carrier dynamics, the response function associated with scattering from the polarization grating can be written, assuming exponential relaxation, as ${ }^{47}$

$$
A_{x y y x}^{(3)}(t)=A_{0}^{(3)} Y_{x y y x}^{(3)} \exp \left(-t / \tau_{\theta}\right) .
$$

Here $\tau_{\theta}$ is the relaxation time associated with momentum randomization, $A_{0}^{(3)}$ is a constant, and $Y_{i j k l}^{(3)}$ is a dimensionless tensor with the symmetry of the third-order susceptibility $\chi_{i j k l}^{(3)}$. The contribution to the change in reflectivity from this response function is given by

$$
\begin{aligned}
& \frac{\Delta R_{\mathrm{PG}}}{R_{s}}(\Delta t)=\int_{-\infty}^{\infty} d t E_{x}^{(p) *}(t-\Delta t) E_{y}^{(e)}(t) \\
& \times \int_{-\infty}^{t} d t^{\prime} A_{x y y x}^{(3)}\left(t-t^{\prime}\right) E_{y}^{(e) *}\left(t^{\prime}\right) E_{x}^{(p)}\left(t^{\prime}-\Delta t\right) .
\end{aligned}
$$

For Gaussian laser pulses and the exponential response function of Eq. (12), the integrals in Eq. (13) can be solved exactly to yield the polarization-grating contribution to the reflectivity

$$
\frac{\Delta R_{\mathrm{PG}}}{R_{s}}(\Delta t)=\frac{\Delta R_{\mathrm{PG}}^{0}}{R_{s}} \exp \left(\frac{-\Delta t^{2}}{\tau_{p}^{2}}\right) .
$$

The parameter $\Delta R_{\mathrm{PG}}^{0} / R_{s}$ is a function of $\tau_{p}$ and $\tau_{\theta}$. Through the term $Y_{x y y x}^{(3)}, \Delta R_{\mathrm{PG}}^{0} / R_{s}$ also depends on the orientation of the sample. However, the time-delay $(\Delta t)$ dependence of Eq. (14) is solely determined by the pulse-width parameter $\tau_{p}$. Thus, this term contains no dynamical infor- mation through its time dependence, but only produces a coherent spike centered at $\Delta t=0$ with a width given by the convolution of the pump and probe pulses.

The second degenerate four-wave mixing contribution to the change in reflectivity arises from the probe beam interacting with the anisotropic component of the pump induced carrier distribution. This contribution is governed by the response function ${ }^{47}$

$$
A_{x x y y}^{(3)}(t)=A_{0}^{(3)}\left(Y_{x x y y}^{(3)}-Y_{x x}^{(1)} Y_{y y}^{(1)}\right) \exp \left(-t / \tau_{\theta}\right) .
$$

Similar to the third-order susceptibility symmetry terms, $Y_{i j}^{(1)}$ has the symmetry of the linear susceptibility $\chi_{i j}^{(1)}$, which for cubic Si is proportional to $\delta_{i j}$, the Kroneker delta. The contribution of this response function to the reflectivity is obtained using Eq. (7) with $A_{\mathrm{FC}}(t)$ replaced by $A_{x x y y}^{(3)}(t)$. Again, the integrals can be analytically solved to yield the anisotropic-distribution contribution to the reflectivity change

$$
\frac{\Delta R_{\mathrm{AD}}}{R_{s}}(\Delta t)=\frac{\Delta R_{\mathrm{AD}}^{0}}{R_{s}}\left[\operatorname{erf}\left(\frac{\Delta \mathrm{t}}{\tau_{\mathrm{p}}}-\frac{\tau_{\mathrm{p}}}{2 \tau_{\theta}}\right)+1\right] \exp \left(\frac{-\Delta t}{\tau_{\theta}}\right) .
$$

As shown below, for our experimental conditions $\tau_{p}$ $\approx \tau_{\theta}$. Even so, Eq. (16) produces a peak with a significant tail that is described by the exponential decay $\exp \left(-\Delta t / \tau_{\theta}\right)$. This contribution thus provides information on carrier momentum relaxation.

In a cubic semiconductor the components of $Y_{i j k l}^{(3)}$ depend upon the orientation of the sample. In our experiment a rotation of the sample about its azimuth can distinguish between the linear-optical effects discussed in Sec. IV A and the nonlinear-optical effects discussed here. For example, if we define $Y_{1221}^{(3)}$ to be $Y_{x y y x}^{(3)}$ when the pump and probe polarizations are oriented along the cubic axis directions of the sample, then upon rotating the crystal through an angle $\phi$ about its azimuth $Y_{x y y x}^{(3)}$ transforms as ${ }^{15}$

$$
\begin{aligned}
Y_{x y y x}^{(3)}(\phi)= & Y_{1221}^{(3)}+\frac{1}{4}[1-\cos (4 \phi)] \\
& \times\left(Y_{1111}^{(3)}-Y_{1122}^{(3)}-Y_{1212}^{(3)}-Y_{1221}^{(3)}\right) .
\end{aligned}
$$

The term $Y_{x x y y}^{(3)}$ transforms similarly. Thus, for the two sample orientations in our experiment $(\phi=0$ and $\phi=\pi / 4)$ we obtain the largest possible differences in the probe intensity associated with $\Delta R_{\mathrm{PG}}$ and $\Delta R_{\mathrm{AD}}$. This difference is clearly observed in the initial spike in the data shown in the lower part of Fig. 1.

\section{DATA ANALYSIS AND DISCUSSION}

\section{A. Momentum and energy relaxation}

Using the sum of Eqs. (8), (10), (14), and (16), along with a model equation for the coherent phonon excitation and decay, ${ }^{32}$ we have fit the short-time-scale data with a leastsquares analysis. In total there are 16 parameters used to analyze the data. There are 10 associated with carrier dynam- 


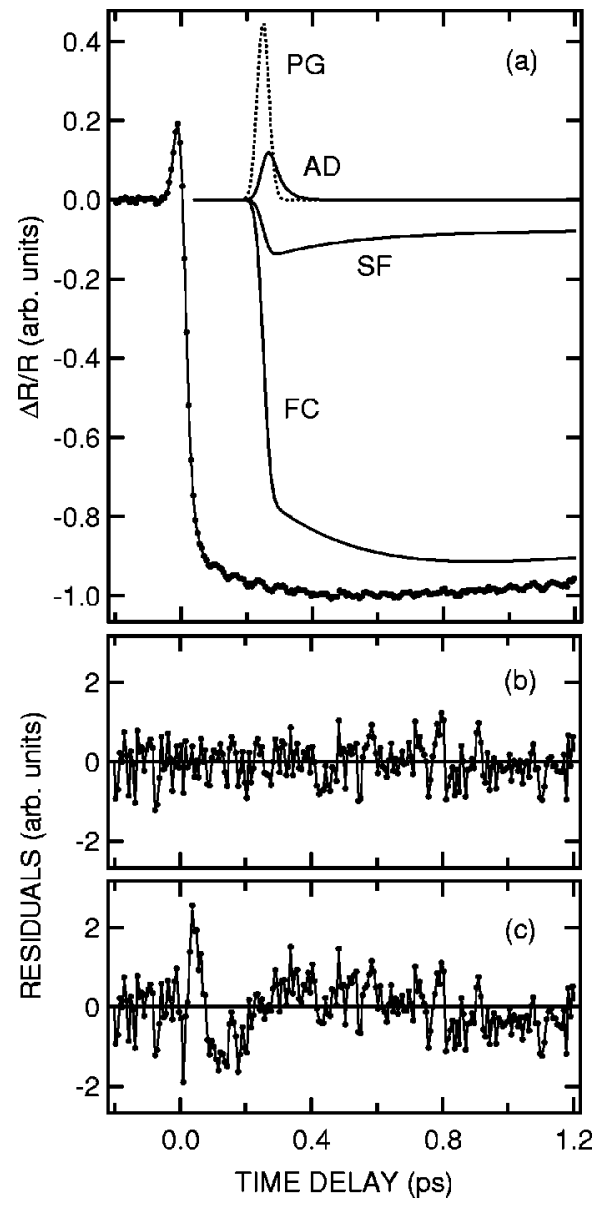

FIG. 4. Least-squares analysis of Si time dependent reflectivity. (a) Data, overall fit, and components [free carrier (FC), state filling (SF), polarization grating (PG), and anisotropic distribution (AD)] are shown. The individual components are shifted by 0.25 ps. (b) Residuals of the fit shown in (a). (c) Residuals of a fit to the data with only the FC, SF, and PG components.

$\operatorname{ics}\left(\Delta R_{\mathrm{FC}}^{\max } / R_{s}, \Delta R_{\mathrm{SF}}^{\max } / R_{s}, \Delta R_{\mathrm{PD}}^{0} / R_{s}, \Delta R_{\mathrm{AD}}^{0} / R_{s}, \tau_{\theta}, \tau_{\mathrm{el}-\mathrm{ph}}\right.$, $\tau_{R}, \Delta m_{\mathrm{opt}}^{*} / m_{300}^{*}, n_{1985} / n_{300}$, and $\left.C_{R}\right), 2$ associated with the laser pulses ( $\tau_{p}$ and the zero-time delay), and four others associated with the coherent-phonon response. ${ }^{32}$ However, because the relaxation of the free-carrier and state-filling responses are characterized by the same relaxation processes, the parameters $\Delta R_{\mathrm{FC}}^{\max } / R_{s}, \Delta R_{\mathrm{SF}}^{\max } / R_{s}, \Delta m_{\mathrm{opt}}^{*} / m_{300}^{*}$, and $n_{1985} / n_{300}$ are highly correlated and cannot all be uniquely determined from the data. We have thus used our calculations to place two constraints on the fitting: we constrain $\Delta m_{\mathrm{opt}}^{*} / m_{300}^{*}$ to the theoretical value of 0.31 and $\Delta R_{\mathrm{SF}}^{\max } / \Delta R_{\mathrm{FC}}^{\max }$ to our calculated value of $\Delta n_{\mathrm{SF}}\left(N_{0}, 300 \mathrm{~K}\right) / \Delta n_{\mathrm{FC}}\left(N_{0}, 300 \mathrm{~K}\right)=0.086$.

A typical least-squares fit with these two constraints is shown in Fig. 4(a). Along with the data (points) and overall fit (solid line through the data), we also display the four individual contributions to the reflectivity (shifted by $0.25 \mathrm{ps}$ for clarity). The statistical nature of the residuals in Fig. 4(b) indicates that the model provides an excellent description of the data. From analysis of all of our data we obtain $\tau_{\theta}=32$ $\pm 5 \mathrm{fs}, \quad \tau_{\mathrm{el}-\mathrm{ph}}=260 \pm 30 \mathrm{fs}, \quad n_{1985} / n_{300}=1.7 \pm 0.3$, and $\tau_{p}$
$=26 \pm 1 \mathrm{fs}$. This extracted value of $\tau_{p}$ is in excellent agreement with the autocorrelator derived value of $25 \pm 1 \mathrm{fs}$. The value of $n_{1985} / n_{300}$ is in very good agreement with our theoretical estimate of 2.1, which suggests that the relative contributions from the free-carrier and state-filling responses are reasonable well described by the constraint $\Delta n_{\mathrm{SF}}\left(N_{0}, 300 \mathrm{~K}\right) / \Delta n_{\mathrm{FC}}\left(N_{0}, 300 \mathrm{~K}\right)=0.086$. However, we emphasize that the values of $\tau_{\theta}$ and $\tau_{\text {el-ph }}$ are largely insensitive to the two imposed constraints. For example, setting the state-filling contribution to zero and using only the freecarrier contribution to describe the linear response results in $\tau_{\text {el-ph }}=240 \pm 30$ fs and no change in $\tau_{\theta}$. Although the contribution to the overall reflectivity from $\Delta R_{\mathrm{AD}} / R_{S}$ is rather small, its inclusion in the analysis is necessary in order to quantitatively describe the reflectivity curve. This is illustrated by the structure present in the residuals if a fit is performed with $\Delta R_{\mathrm{AD}}^{0} / R_{S}$ constrained to zero, as shown in Fig. $4(\mathrm{c})$.

Our value of $32 \pm 5$ fs for $\tau_{\theta}$ is, to our knowledge, the first ultrafast experimental determination of this parameter. Because of lower time resolution, previous investigations have only suggested the time scale for this parameter. The degenerate four-wave mixing experiments of Buhleier et al. with $2.0 \mathrm{eV}, 100 \mathrm{fs}$ pulses indicated momentum relaxation on a time scale of $10 \mathrm{fs} .{ }^{15}$ The transient grating experiments of Sjodin et al. with $1.55 \mathrm{eV}, \sim 100$ fs pulses also showed that the momentum reorientation time is significantly less that $100 \mathrm{fs}^{24}$

Theoretical calculations suggest that carrier-phonon scattering slightly dominates the carrier-carrier scattering contribution to $\tau_{\theta}$. Theoretical electron-phonon and hole-phonon scattering times have been calculated by a number of researchers that use Monte Carlo simulations to model hotcarrier transport. For an excess carrier energy of $0.3 \mathrm{eV}$ carrier-phonon scattering times range between 20 and 60 fs. ${ }^{2,3,26}$ Carrier-carrier scattering rates for carrier densities and temperatures appropriate to our experiment have been calculated in a series of papers by Combescot et al. and Sernelius. ${ }^{25,49}$ From their theoretical expressions we calculate electron-electron and electron-hole scattering times of 155 and $170 \mathrm{fs}$, respectively, for our initial excitation density and excess carrier energy. ${ }^{50}$ Combining these scattering rates (assuming that they are independent) yields an overall carrier-carrier scattering time of $80 \mathrm{fs}$. Combining this rate with the range of theoretical carrier-phonon rates yields an overall relaxation time between 16 and 35 fs, entirely consistent with our measured value of $\tau_{\theta}=32 \pm 5 \mathrm{fs}$.

The carrier energy-relaxation time $\tau_{\mathrm{el}-\mathrm{ph}}$ is an important parameter in Monte Carlo modeling of semiconductor devices. ${ }^{1-4}$ Our result, $\tau_{\text {el-ph }}=260 \pm 30 \mathrm{fs}$, is most directly comparable to the result of Sjodin et al., who obtain an average value of $240 \pm 30 \mathrm{fs}$ for excitation densities less than $\sim 10^{20} \mathrm{~cm}^{-1} .{ }^{14}$ Our result also compares favorably with a dc-transport inferred value of $270 \mathrm{fs} .{ }^{51}$ For comparison with Monte Carlo derived values of $\tau_{\text {el-ph }}$ we have plotted both experimental and theoretical values of $\tau_{\text {el-ph }}$ as a function of excess carrier energy in Fig. 5. Experimental data from three previous studies, ${ }^{8,9,14}$ in addition to the present study, are represented by the solid symbols and solid line (the solid line 


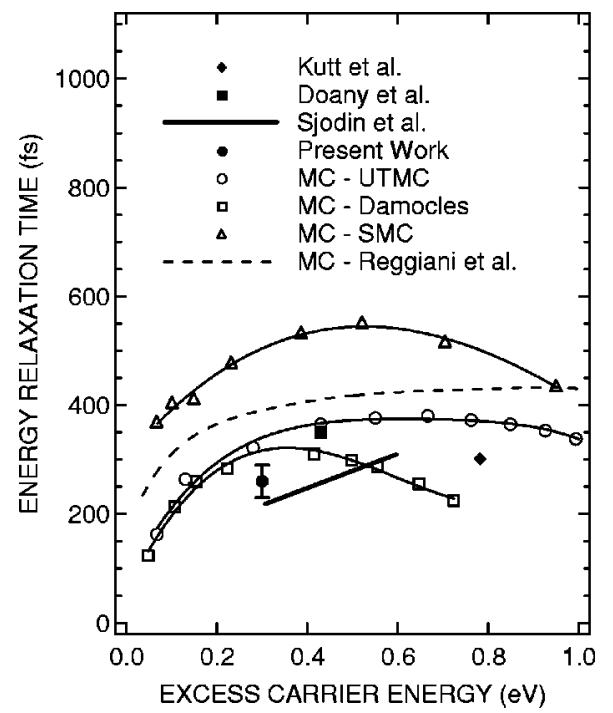

FIG. 5. Comparison of experimental and theoretical values of $\tau_{\text {el }-p h}$. Calculated values are for electron cooling (dashed curve) and hole cooling (open symbols and thin solid curves). Experimental values are represented by solid symbols and the thick solid line.

is a linear fit to the data of Sjodin et al. ${ }^{14}$ ). In determining the excess carrier energy for the experimental values of $\tau_{\text {el-ph }}$ both linear $^{29}$ and two-photon absorption ${ }^{9,42}$ have been included. From the study of Sjodin et al. we have only used data at carrier densities less than $\sim 2 \times 10^{20} \mathrm{~cm}^{-3}$, above which intracarrier screening affects the carrier-phonon interaction. ${ }^{52}$ Monte Carlo calculations of both the hole energy decay time (open symbols with dotted lines) and electron energy decay time (dashed line) are also shown. ${ }^{4,53}$ The comparison between experimental and theoretical values of $\tau_{\mathrm{el}-\mathrm{ph}}$ indicates that the UTMC and Damocles Monte Carlo codes $^{4}$ produce values for $\tau_{\text {el-ph }}$ that are closest to the experimental data. We also note that a very recent measurement indicates $\tau_{\mathrm{el}-\mathrm{ph}}=240 \mathrm{fs}$ for nanocrystalline $\mathrm{Si}^{54}$

There have been several ultrafast photoemission studies of the $\mathrm{Si}(001) 2 \times 1$ surface. ${ }^{20-23}$ In principle, these measurements are able to follow both intraelectron thermalization and hot-electron cooling by measuring the time dependence of the electron distribution function. Indeed, using $2.0 \mathrm{eV}$, $\sim 120$ fs pump pulses Goldman and Prybyla observed thermalized electron distributions characterized by temperatures of 1200 and $800 \mathrm{~K}$ at 120 and 180 fs delay times, respectively. Their results indicate that electron-electron thermalization (and hence electron-electron scattering) is significantly faster than $\sim 100 \mathrm{fs}$ and that $\tau_{\mathrm{el}-\mathrm{ph}}$ is $\sim 90 \mathrm{fs}{ }^{21} \mathrm{In}$ contrast, the photoemission data of Jeong and Bokor, obtained with $1.55 \mathrm{eV}, 150 \mathrm{fs}$ pump pulses, are consistent with an intraelectron thermalization time of a few hundred fs and a value for $\tau_{\text {el-ph }}$ of $400 \mathrm{fs}^{23}$ The discrepancy among results from the two photoemission studies and the present study can be explained by the surface-sensitive nature of the photoemission process, ${ }^{20,23}$ which for $\mathrm{Si}(001) 2 \times 1$ is dominated by electrons photoemitted from defect-induced surface states. ${ }^{23}$ Thus, in the photoemission measurements surfacestate dynamics obscure bulk-carrier dynamics so that measured relaxation times cannot be directly associated with bulk scattering processes. It is therefore not surprising that photoemission inferred values for carrier-carrier and carrierphonon scattering processes are somewhat different than those from our bulk-sensitive measurements. Furthermore, even though the two photoemission studies were of nominally the same $\operatorname{Si}(001) 2 \times 1$ surface, the observed differences can be attributed to differences in surface defect density on the measured $\mathrm{Si}$ surfaces. ${ }^{23}$

\section{B. Carrier recombination}

The recovery of the reflectivity signal shown in 120 ps scans of Fig. 2 is predominantly due to the reduction in excited carrier density $N$ as electrons and holes recombine across the Si band gap. Both the free-carrier [Eq. (5)] and state-filling contributions to the reflectivity diminish with recombination. For our samples the recombination process is dominated by Shockley-Reed-Hall (SRH) recombination involving surface band-gap states. ${ }^{11}$ In the SRH recombination process the electron-hole energy $(\sim 1.2 \mathrm{eV}$ per electron-hole pair) is absorbed by the lattice via multiphonon emission. This energy return to the lattice can produce a non-negligible contribution to the reflectivity change from the associated increase in lattice temperature $T_{L}$ [Eq. (11)] near the sample surface. ${ }^{11}$

In order to describe the time dependence of $N$ and $T_{L}$ appropriate to the time scale of Fig. 2 we use a model that includes diffusion of both carrier density $N$ and lattice temperature $T_{L} \cdot{ }^{10,11,17}$ The diffusion equations for $N$ and $T_{L}$ are coupled through boundary conditions that depend upon the surface recombination velocity $S$, which describes the rate of SRH recombination via surface states. The recombination velocity $S$, the only free parameter in the diffusion model, is proportional to effective surface-state trap density $n_{S}$ and cross section $\sigma$, and carrier thermal velocity $v_{\text {th }}$ $\approx 10^{7} \mathrm{~cm} \mathrm{~s}^{-1} ; S \approx \sigma v_{\text {th }} n_{S} .55$ If we assume that $\sigma \approx 1 / n_{A}$, where $n_{A}=6.8 \times 10^{14} \mathrm{~cm}^{-2}$ is the surface-atom density on the $\operatorname{Si}(001)$ surface, then $S \approx v_{\text {th }} n_{S} / n_{A}$. Because the electrons and lattice can be considered to be in thermal equilibrium, the relative contributions to $\Delta R_{S} / R_{S}$ from $N$ and $T_{L}$ can be determined from $\Delta n_{\mathrm{FC}}\left(N, T_{L}\right), \Delta n_{\mathrm{SF}}\left(N, T_{L}\right)$, and $\Delta n_{\mathrm{LT}}\left(T_{L}\right)$, as described in Sec. IV A.

Using this model we have numerically solved the diffusion equation for the time dependence of near-surface values of $N$ and $T_{L}$. In Fig. 6 we show calculated time-dependent reflectivity changes that illustrate the separate reflectivity contributions from $\Delta N$ and $\Delta T_{L}$ for $S=3 \times 10^{4}, S=3$ $\times 10^{5}$, and $S=3 \times 10^{6} \mathrm{~cm} \mathrm{~s}^{-1}$. Note that for a large enough recombination velocity the $T_{L}$ contribution can dominate the $N$ contribution and cause the reflectivity to initially recover past its initial value. We have previously observed this behavior on other Si surfaces. ${ }^{11}$

In Fig. 2(b) we compare the experimental reflectivity curves with calculated reflectivity curves. In each comparison $S$ has been adjusted to produce the best overall match to the experimental data. Values of $S$ used in the three calculated curves in Fig. 2(b) are $2.0 \times 10^{4}, 3.4 \times 10^{4}$, and 4.6 $\times 10^{4} \mathrm{~cm} \mathrm{~s}^{-1}$. Whereas the decrease in $N$ on the few-ps time scale of Figs. 1 and 4 can be described by an exponential 


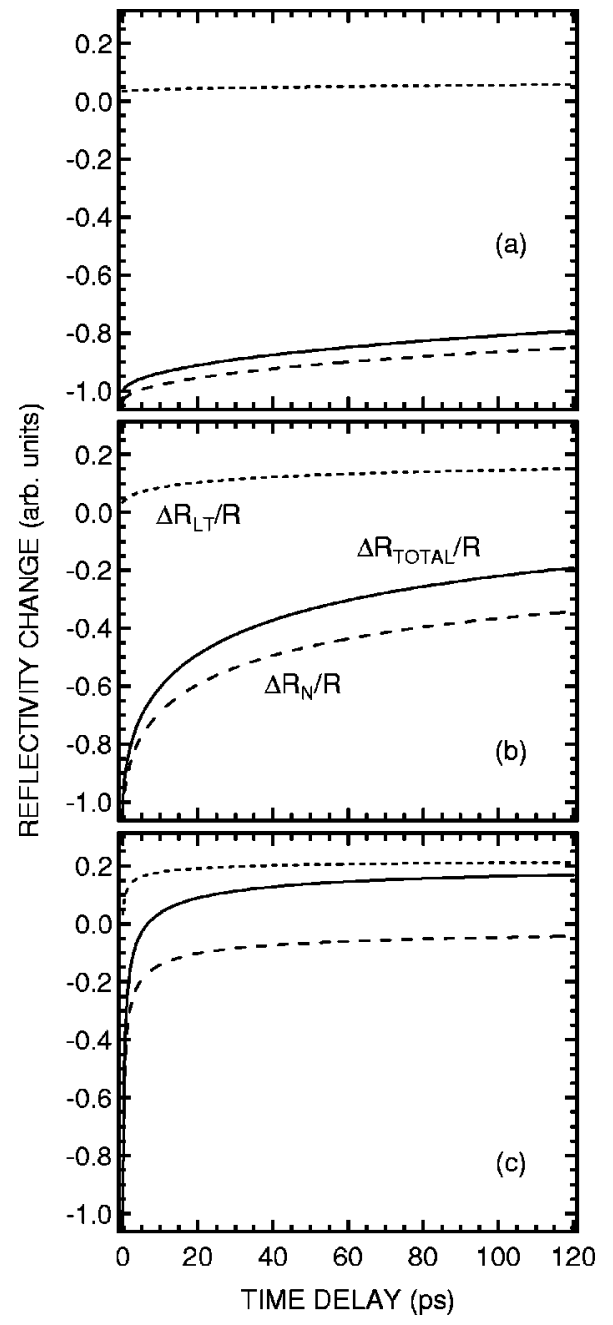

FIG. 6. Calculated time-dependent reflectivity curves for Si (solid lines) using carrier and temperature diffusion model (see text). Carrier (free carrier and state filling) and lattice-temperature contributions are shown as long-dashed and short-dashed curves, respectively. (a) $S=3 \times 10^{4} \mathrm{~cm} \mathrm{~s}^{-1}$. (b) $S=3 \times 10^{5} \mathrm{~cm} \mathrm{~s}^{-1}$. (c) $S=3 \times 10^{6} \mathrm{~cm} \mathrm{~s}^{-1}$.

decay, the data of Fig. 2 and the results of the diffusionmodel calculation show that on a 120 -ps time scale the recovery in reflectivity is relatively faster at short times and slower at long times than a simple exponential decay. Additionally, the comparison in Fig. 2(b) between the calculations and data shows that the measured short-time change is relatively faster than short-time change calculated in the model. This is likely due to two effects. ${ }^{19,23}$ First, the recombination velocity is expected to have some time dependence due to transient occupation effects of the surface states. ${ }^{20}$ Additionally, surface-charge induced band bending is expected to drive either electrons or holes away from the surface, decreasing the effective recombination velocity as a function of time. Neither of these effects are included in the diffusion model. For all of our native-oxide covered samples we extract a value for $S$ of $(3 \pm 1) \times 10^{4} \mathrm{~cm} \mathrm{~s}^{-1}$. Using $S$ $\approx v_{\text {th }} n_{s} / n_{A}$, this value of $S$ corresponds to a surface-state trap density of $(0.3 \pm 0.1) \times 10^{-2}$ monolayer for native-oxide terminated $\mathrm{Si}(001)$.

\section{SUMMARY}

We have studied bulk carrier dynamics near native-oxide covered $\mathrm{Si}(001)$ using ultrafast pump-probe reflectivity . From the measurements we have determined the carrier momentum and energy relaxation time scales. The momentum relaxation time $\tau_{\theta}=32 \pm 5 \mathrm{fs}$ is the first measurement of this parameter in Si. Both carrier-phonon and carrier-carrier scattering appear to contribute to momentum relaxation. The carrier energy relaxation time $\tau_{\text {el-ph }}=260 \pm 30$ fs agrees well with previous experimental determination of this parameter. The experimental values of $\tau_{\text {el-ph }}$ agree reasonably well with recent Monte Carlo derived values. Finally, we have extracted a value for the surface recombination velocity of native-oxide terminated $\mathrm{Si}(001)$, which is directly related to the density of traps that participate in surface recombination.

\section{APPENDIX}

In this appendix we discuss the reflectivity from an inhomogeneously excited solid, such as occurs in the experiments discussed here. In particular, we study the reflectivity of a solid with a dielectric function that varies exponentially with depth $z$ into the solid as

$$
\hat{\varepsilon}(z)=\hat{\varepsilon}_{0}-\hat{\eta} \exp (-z / \delta)
$$

Here $\delta$ describes the decay length for relaxation of the dielectric function to the bulk dielectric $\varepsilon_{0}$ and $-\hat{\eta}$ is the change in $\hat{\varepsilon}$ at the surface (so that $\hat{\varepsilon}_{0}-\hat{\eta}$ is the dielectric function near the surface). The reflectivity for the two limits of large and small $\delta$ are fairly easy to surmise. If $\delta$ is much smaller than some appropriate length scale (which as we show below is related to the wavelength of the probe radiation inside the solid) so that the changes in optical constants are confined to only a very small spatial region near the surface, then we expect the reflectivity to be characterized by the background dielectric function $\varepsilon_{0}$. Conversely, if $\delta$ is large, then we expect the reflectivity to be characterized by $\varepsilon_{0}-\eta$, the dielectric function near the surface. We show here that the transition in reflectivity between these two limits occurs for $\delta=d_{\text {obs }}$, where $d_{\mathrm{obs}}=\lambda /\left(4 \pi\left|\hat{n}_{0}\right|\right)$. The parameter $d_{\text {obs }}$ is known as the effective observation depth of the probe radiation. This length can often be much smaller than the penetration depth of the probe radiation $\delta_{\text {probe }}$ $=\lambda /\left(4 \pi \kappa_{0}\right)$. However, as we show below, it is the ratio $\delta / d_{\text {obs }}$ that defines the transition between the two reflectivity extremes rather than the ratio $\delta / \delta_{\text {probe }}$. The normal-incidence reflectivity has previously been discussed in the literature: Vakhnenko and Strizhevskii has solved this problem exactly. ${ }^{56}$ However, the exact solution involves nonintegralorder Bessel functions. Here we outline a perturbation-theory solution that provides much simpler expressions for calculating the reflectivity.

Assuming linear polarization, normal incidence, and harmonic time dependence the wave equation for the electric field $E(z)$ inside the solid can be written as 


$$
\frac{d^{2} E(z)}{d z^{2}}+k^{2} \hat{\varepsilon}(z) E(z)=0,
$$

where $k=\omega / c$ and $\hat{\varepsilon}(z)$ is given by Eq. (A1). The solution for the electric field is found using perturbation theory, ${ }^{57}$ where the small parameter in the problem is $\hat{\eta} / \hat{\varepsilon}_{0}$. (For our experiments $\left|\hat{\eta} / \hat{\varepsilon}_{0}\right| \approx 0.02$.) The solution to Eq. (A2) to first order in $\hat{\eta} / \hat{\varepsilon}_{0}$ is

$$
E(z)=E_{0} \exp \left(i k \hat{n}_{0} z\right)\left\{1+\frac{\hat{\eta} k^{2} \delta^{2}}{1-i \delta / \hat{d}_{\mathrm{obs}}} \exp (-z / \delta)\right\},
$$

where $\hat{d}_{\mathrm{obs}}=1 /\left(4 \pi \hat{n}_{0}\right)$. The normal-incidence reflectivity $R$ can be expressed as

$$
R=\left|\frac{1-\hat{z}_{s}}{1+\hat{z}_{s}}\right|^{2}
$$

where

$$
\hat{z}_{s}=\left.i k \frac{E}{d E / d z}\right|_{z=0}
$$

is the normalized surface impedance. Substituting Eq. (A3) into Eq. (A5) and keeping terms up to linear order in $\hat{\eta} / \hat{\varepsilon}_{0}$ yields

$$
\hat{z}_{s}=\hat{z}_{s 0}\left(1+\frac{\hat{\eta}}{2 \hat{\varepsilon}_{o}} \frac{1}{1+i \hat{d}_{\mathrm{obs}} / \delta}\right),
$$

where $\hat{z}_{s 0}$ is the surface impedance for $\hat{\eta}=0$. Equation (A6) has the expected limits for small and large $\delta$. For $\delta / d_{\text {obs }}$ $\ll 1, \hat{z}_{s} \approx \hat{z}_{s 0}$, which is equivalent to the surface impedance for a uniform material with $\hat{\varepsilon}=\hat{\varepsilon}_{0}$, while for $\delta / d_{\mathrm{obs}} \gg 1, \hat{z}_{s}$ $\approx \hat{z}_{s 0}\left[1+\hat{\eta} /\left(2 \hat{\varepsilon}_{0}\right)\right]$, which is equivalent to the surface impedance for a uniform material with $\hat{\varepsilon}=\hat{\varepsilon}_{0}-\hat{\eta}$.

Using Eq. (A6) in Eq. (A4) we have calculated the reflec-

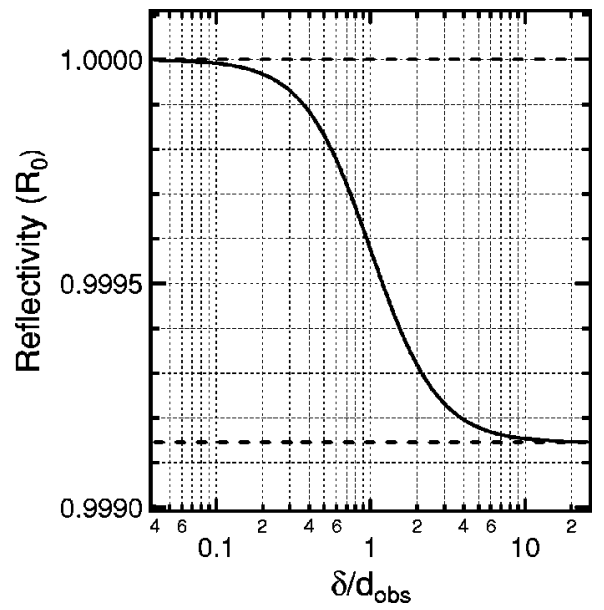

FIG. 7. Normalized Si reflectivity versus $\delta / d_{\text {obs }}$. Solid curve corresponds to $\hat{\varepsilon}_{0}=13.656+i 0.048$ and $\hat{\eta}=0.02$. Upper dashed line corresponds to a uniform solid with $\hat{\varepsilon}=\hat{\varepsilon}_{0}$. Lower dashed curve corresponds to a uniform solid with $\hat{\varepsilon}=\hat{\varepsilon}_{0}-\hat{\eta}$.

tivity $R$ (normalized by the reflectivity $R_{0}$ for $\hat{\eta}=0$ ) as a function of $\delta$ for conditions appropriate to the change in $\hat{\varepsilon}$ in our experiment: we use $\hat{\varepsilon}_{0}=13.656+i 0.048$ and $\hat{\eta}=0.02$. The results are plotted in Fig. 7. As can be seen in the graph, for small $\delta / d_{\mathrm{obs}}$ the reflectivity approaches $R_{0}$, while for large $\delta / d_{\text {obs }}$ the reflectivity approaches a constant value, which is appropriate to $\hat{\varepsilon}=\hat{\varepsilon}_{0}-\hat{\eta}$. Note that for $\delta / d_{\text {obs }}=1$ the reflectivity is exactly half way between the reflectivity for the small and large $\delta / d_{\text {obs }}$ limits. For the initial excitation conditions in our experiment $\delta / d_{\mathrm{obs}}=570$. Hence, initially the probe-pulse reflectivity is characterized by the nearsurface dielectric function. At longer times, carrier and temperature diffusion produce a rather complicated $z$-dependence to $\hat{\varepsilon}{ }^{11}$ However, after approximately $1.5 \mathrm{ps}$ the length scale for the change in $\hat{\varepsilon}$ near the surface is greater than $5 d_{\text {obs }}$. Thus, in modeling the 120-ps reflectivity changes we also only need to consider the near-surface dielectric function.
${ }^{1}$ M. V. Fischetti and S. E. Laux, Phys. Rev. B 38, 9721 (1988); T. Kunikiyo, et al., J. Appl. Phys. 75, 297 (1994); B. Fischer and K. Hofmann, Appl. Phys. Lett. 76, 583 (2000).

${ }^{2}$ X. Wang, V. Chandramouli, C. M. Maziar, and A. F. Tasch, J. Appl. Phys. 73, 3339 (1993).

${ }^{3}$ M. V. Fischetti, S. E. Laux, and E. Crabbé, J. Appl. Phys. 78, 1058 (1995).

${ }^{4}$ S. Jallepalli, M. Rashed, W. K. Shih, C. M. Maziar, and A. F. Tasch, J. Appl. Phys. 81, 2250 (1997).

${ }^{5}$ M.-C. Cheng, R. Chennupati, and Y. Wen, J. Appl. Phys. 78, 4490 (1995).

${ }^{6}$ M. C. Downer and C. V. Shank, Phys. Rev. Lett. 56, 761 (1986).

${ }^{7}$ A. Esser, W. Kütt, M. Strahnen, G. Maidhorn, and H. Kurz, Appl. Surf. Sci. 46, 446 (1990); F. E. Doany, D. E. Grischkowsky, and C.-C. Chi, Appl. Phys. Lett. 50, 460 (1987); A.
Chin, K. Y. Lee, B. C. Lin, and S. F. Horng, ibid. 69, 653 (1996).

${ }^{8}$ F. E. Doany and D. E. Grischkowsky, Appl. Phys. Lett. 52, 36 (1988).

${ }^{9}$ W. Kütt, A. Esser, K. Seibert, U. Lemmer, and H. Kurz, Proc. SPIE 1268, 154 (1990).

${ }^{10}$ T. Tanaka, A. Harata, and T. Sawada, J. Appl. Phys. 82, 4033 (1997).

${ }^{11}$ A. J. Sabbah and D. M. Riffe, J. Appl. Phys. 88, 6954 (2000).

${ }^{12}$ T. Tanaka, A. Harata, and T. Sawada, Jpn. J. Appl. Phys. 35, 3642 (1996).

${ }^{13}$ C. M. Li, Z. C. Ying, T. Sjodin, and H.-L. Dai, Appl. Phys. Lett. 66, 3501 (1995); C. M. Li, T. Sjodin, and H.-L. Dai, Phys. Rev. B 56, 15252 (1997).

${ }^{14}$ T. Sjodin, H. Petek, and H.-L. Dai, Phys. Rev. Lett. 81, 5664 (1998). 
${ }^{15}$ R. Buhleier, G. Lüpke, G. Marowsky, Z. Gogolak, and J. Kuhl, Phys. Rev. B 50, 2425 (1994).

${ }^{16}$ J. E. Rothenberg, Opt. Lett. 13, 713 (1980).

${ }^{17}$ O. B. Wright and V. E. Gusev, Appl. Phys. Lett. 66, 1190 (1995).

${ }^{18}$ J. Bokor, R. Storz, R. R. Freeman, and P. H. Bucksbaum, Phys. Rev. Lett. 57, 881 (1986).

${ }^{19}$ N. J. Halas and J. Bokor, Phys. Rev. Lett. 62, 1679 (1989).

${ }^{20}$ M. W. Rowe, H. Liu, G. P. Williams, and R. T. Williams, Phys. Rev. B 47, 2048 (1993).

${ }^{21}$ J. R. Goldman and J. A. Prybyla, Phys. Rev. Lett. 72, 1364 (1994).

${ }^{22}$ S. Jeong, H. Zacharias, and J. Bokor, Phys. Rev. B 54, R17 300 (1996).

${ }^{23}$ S. Jeong and J. Bokor, Phys. Rev. B 59, 4943 (1999).

${ }^{24}$ T. Sjodin, C. M. Li, H. Petek, and H.-L. Dai, Chem. Phys. 251, 205 (2000).

${ }^{25}$ B. E. Sernelius, Phys. Rev. B 40, 12438 (1989).

${ }^{26}$ Y. Kamakura, I. Kawashima, K. Deguchi, and K. Taniguchi, J. Appl. Phys. 88, 5802 (2000).

${ }^{27}$ M. T. Asaki et al., Opt. Lett. 18, 977 (1993).

${ }^{28}$ D. M. Riffe and A. J. Sabbah, Rev. Sci. Instrum. 69, 3099 (1998).

${ }^{29}$ D. E. Aspnes and A. A. Studna, Phys. Rev. B 27, 985 (1983).

${ }^{30}$ S. M. Sze, Physics of Semiconductor Devices (Wiley, New York, 1981).

${ }^{31}$ T. Miura, M. Niwano, D. Shoji, and N. Miyamoto, J. Appl. Phys. 79, 4373 (1996).

${ }^{32}$ A. J. Sabbah, M. Holtz, and D. M. Riffe (unpublished).

${ }^{33}$ R. Scholz, T. Pfeifer, and H. Kurz, Phys. Rev. B 47, 16229 (1993).

${ }^{34}$ This excess energy is calculated taking into account both one and two photon absorption. Because the absorption is indirect (phonon-assisted) the hot holes and hot electrons are expected to equally share the excess energy.

${ }^{35}$ The effects of band nonparabolicity are included in calculating the hot-carrier temperature from the excess carrier energy.

${ }^{36}$ H. M. van Driel, Appl. Phys. Lett. 44, 617 (1984).

${ }^{37}$ D. M. Riffe, J. Opt. Soc. Am. B 19, 1092 (2002).

${ }^{38}$ F. Stern, Phys. Rev. 133, A1653 (1964).

${ }^{39}$ G. Grosso and G. P. Parravicini, Solid State Physics (Academic Press, New York, 2000).

${ }^{40}$ G. E. Jellison and H. H. Burke, J. Appl. Phys. 60, 841 (1986).

${ }^{41}$ B. R. Bennett, R. A. Soref, and J. A. del Alamo, IEEE J. Quantum Electron. 26, 113 (1990).
${ }^{42}$ D. H. Reitze, T. R. Zhang, W. M. Wood, and M. C. Downer, J. Opt. Soc. Am. B 7, 84 (1990).

${ }^{43}$ K. Sokolowski-Tinten and D. von der Linde, Phys. Rev. B 61, 2643 (2000).

${ }^{44}$ P. A. Wolff, Phys. Rev. 126, 405 (1962); K.-F. Berggren and B. E. Sernelius, Phys. Rev. B 24, 1971 (1981); R. Zimmerman, Phys. Status Solidi B 146, 371 (1988).

${ }^{45}$ C. V. Shank and D. H. Auston, Phys. Rev. Lett. 34, 479 (1975); Z. Vardeny and J. Tauc, Opt. Commun. 39, 396 (1981); W. Z. Lin, L. G. Fujimoto, E. P. Ippen, and R. A. Logan, Appl. Phys. Lett. 50, 124 (1987); W. Z. Lin, L. G. Fujimoto, E. P. Ippen, and R. A. Logan, ibid. 51, 161 (1987); C. J. Kennedy et al., Phys. Rev. Lett. 32, 419 (1974); Z. Vardeny and J. Tauc, ibid. 46, 1223 (1981).

${ }^{46}$ J. R. Lindle, S. C. Moss, and A. L. Smirl, Phys. Rev. B 20, 2401 (1979).

${ }^{47}$ B. S. Wherrett, A. L. Smirl, and T. F. Boggess, IEEE J. Quantum Electron. QE-19, 680 (1983).

${ }^{48}$ A. L. Smirl, T. F. Boggess, B. S. Wherrett, G. P. Perryman, and A. Miller, IEEE J. Quantum Electron. QE-19, 690 (1983).

${ }^{49}$ M. Combescot and R. Combescot, Phys. Rev. B 35, 7986 (1987); N. S. Wingreen and M. Combescot, ibid. 40, 3191 (1989); B. E. Sernelius, Phys. Rev. B 43, 7136 (1991).

${ }^{50}$ The theoretical carrier-carrier scattering rates have been calculated for a well defined carrier temperature. Obviously the carrier thermalization time must be somewhat longer than the carrier-carrier scattering rate, so that these calculations do not directly apply to the prethermalized state of the carriers. However, because the carrier distribution is nondegenerate, we do not expect the carrier-carrier scattering rates to depend significantly on the carrier distribution function for a given excess carrier energy.

${ }^{51}$ D. Sinitsky, F. Assaderaghi, M. Orshansky, J. Bokor, and C. Hu, Solid-State Electron. 41, 1119 (1997).

${ }^{52}$ E. J. Yoffa, Phys. Rev. B 21, 2415 (1980); 23, 1909 (1981).

${ }^{53}$ E. Constant, in Hot-Electron Transport in Semiconductors, edited by L. Reggiani (Springer-Verlag, New York, 1985), p. 227.

${ }^{54}$ K. E. Myers, Q. Wang, and S. L. Dexheimer, Phys. Rev. B 64, 161309 (2001).

${ }^{55}$ D. K. Schroder, IEEE Trans. Electron Devices 44, 160 (1997).

${ }^{56}$ I. F. Vakhnenko and V. L. Strizhevskii, Sov. Phys. Semicond. 3, 1562 (1970).

${ }^{57}$ C. M. Bender and S. A. Orzag, Advanced Mathematical Methods for Scientists and Engineers (McGraw-Hill, New York, 1978). 